\title{
Field-dependent magnetic anisotropy in a single-ion magnet measured using polarized neutron powder diffraction
}

\author{
E. A. Klahn ${ }^{1}$, A. M. Thiel ${ }^{1}$, I. A. Kibalin, ${ }^{2}$ A. Gukasov ${ }^{2}$, J. Overgaard ${ }^{1}$ \\ ${ }^{1}$ Dept. of Chemistry, Aarhus University, Langelandsgade 140, 8200 Aarhus N, Denmark, ${ }^{2}$ Laboratorie Léon Brillouin, CEA-CNRS, \\ CE-Saclay, 91191 Gif-sur-Yvette, France \\ eklahn@chem.au.dk
}

Single-ion magnets (SIMs) are a class of metal-organic coordination complexes with the intriguing ability to sustain a magnetic moment after the removal of a magnetizing field [1]. This ability originates in orbital angular momentum of unpaired electrons, introducing magnetic anisotropy that leads to a barrier to reversal of the SIM magnetic moment. Magnetic anisotropy is therefore a key property in the search for new and improved SIMs, and it is imperative to be able to measure magnetic anisotropy experimentally.

In 2002, it was shown that polarized neutron diffraction from single crystals (PND) could be used to obtain information on so called ionic site susceptibilities [2], which are tensor quantities that show the response of the magnetic moment of the ion to an external magnetic field. Site susceptibilities give direct access to the magnetic anisotropy of a compound, and we have earlier used this technique to measure the magnetic anisotropy of both lanthanide and transition metal SIMs in single crystals [3, 4]. Importantly, the technique was recently extended for application to powder samples [5].

Utilizing this exciting development, we have performed polarized neutron powder diffraction (pPND) on the $\mathrm{SIM} \mathrm{CoCl}_{2}(\mathrm{tmtu})_{2}$, tmtu=tetramethylthiourea (1). The compound shows zero-field splitting and slow relaxation of its magnetic moment [6], both requirements for a SIM. With pPND we obtain the orientation of the magnetic anisotropy with respect to the molecular structure (Fig. 1), and follow its dependence on magnetic field strength, directly from a powder sample. Comparison with PND measured on a single crystal of the Br-analogue $\mathrm{CoBr}_{2}(\mathrm{tmtu})_{2}(2)$ shows that the powder and single crystal techniques give comparable results.

In this contribution, I will discuss the site susceptibility model, its application to both single crystal and powder samples and the magneto-structural correlations obtained from these measurements. The extension of the technique to powders entails that compounds can be studied, for which growth of single crystals for neutron diffraction is unattainable. This opens the possibility for magnetic anisotropy studies on a much wider range of molecular magnetic compounds under a larger range of experimental conditions.
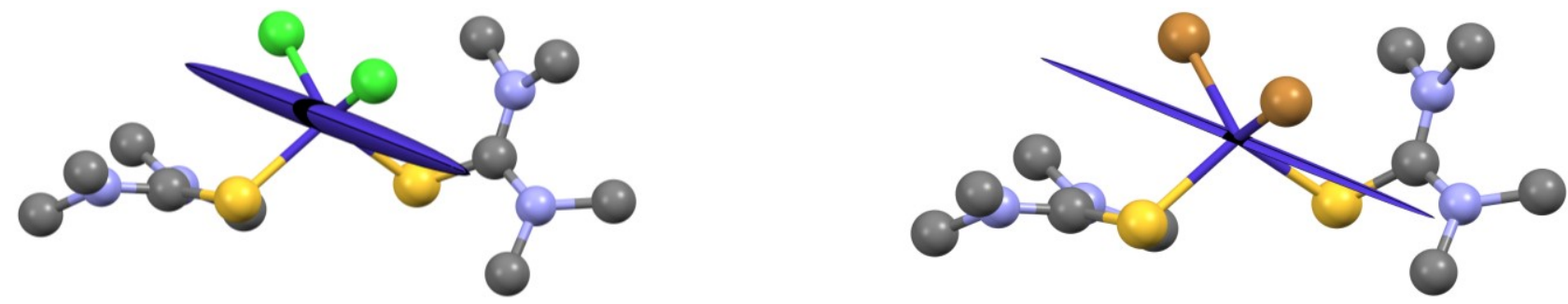

Figure 1. Left: Site susceptibility of $\mathbf{1}$ at $2 \mathrm{~K}, 1 \mathrm{~T}$ measured using polarized neutron powder diffraction and plotted on top of the molecular structure. Right: Site susceptibility of 2, measured at $3 \mathrm{~K}, 1 \mathrm{~T}$ using single crystal polarized neutron diffraction. The site susceptibilities have been scaled arbitrarily to fit the molecular structure. Atoms are Co (purple), S (yellow), N (blue), C (gray), Cl (green), Br (brown). Hydrogens have been omitted.

[1] Villain, J., Gatteschi, D., Sessoli, R. (2006). Molecular nanomagnets. Oxford: Oxford University Press.

[2] Gukasov, A., Brown, J. P. (2002). J. Phys.: Condens. Matter. 14, 8831.

[3] Klahn, E.A., Gao, C., Gillon, B., Gukasov, A., Fabrèges, X., Piltz, R.O., Jiang, S.-D., Overgaard, J. (2018). Chem. Eur. J. 24 , 16576.

[4] Tripathi, S., Vaidya, S., Ahmed, N., Klahn, E. A., Cao, H., Spillecke, L., Koo, C., Spachmann, S., Klingeler, R., Rajaraman, G., Overgaard, J., Shanmugam, M. (2021). Cell Rep. Phys. Sci. 2, 100404.

[5] Kibalin, I. A., Gukasov, A. (2019). Physical Review Research. 1, 033100.

[6] Vaidya, S., Shukla, P., Tripathi, S., Rivière, E., Mallah, T., Rajaraman, G., Shanmugam, M. (2018). Inorg. Chem. $57,3371$.

Keywords: powder diffraction; magnetic anisotropy; polarized neutrons; single-molecule magnets

Acta Cryst. (2021), A77, C346 